1l-point scale for infants, which incorporates ocular responses (ranging from normal pursuit to fixed pupils), verbal responses (ranging from crying to apnea), and motor responses (from flexion/ extension to flaccidity). A uniformly tested and accepted coma scale for infants and children would be advantageous. Coma scores as low as 3-4 carry the same prediction of poor outcome at any age; coma scores of 5-8 may carry a less serious significance in young children than in adults.

\title{
ALPHA COMA IN THE EEG
}

Recorded EEG rhythms within the alpha-frequency band, paradoxically resembling waking patterns but in apparently comatose patients, a pattern termed alpha coma, have been reviewed over a $10 \mathrm{yr}$ period in the Division of Electroencephalography, University of Washington School of Medicine, Seattle, WA. Of 50 patients with records showing the alpha-pattern coma, 49 were admitted with cardio-pulmonary arrest and one had developed alpha coma with hyperglycemic, hyperosmolar coma. In addition to the alpha activity, arrhythmic delta waves were present diffusely in $25 \%$ and theta waves in 23\%. The outcomes of patients with or without alpha coma after cardiac arrest did not differ significantly, the majority not regaining consciousness and dying in hospital. The single patient with hyperglycemic coma regained consciousness and was discharged but did not fully recover cognitive function. A review of the literature did not preclude neurological recovery following alpha coma. (Austin EJ et al. Etiology and prognosis of alpha coma. Neurology May 1988; 38: 773-777).

COMMENT: Alpha coma usually follows cardiac arrest and can be identified in $25 \%$ of patients. It also follows brainstem lesions, sedative drug overdose, respiratory arrest, and severe disturbance of glucose metabolism. Where alpha coma follows a condition other than cardiac arrest or a brain stem lesion the outcome is usually good. The prognosis is especially favorable after drug overdose.

\section{MOVEMENT DISORDERS}

\section{DYSTONIA AND BIOPTERIN DEFICIENCY}

Five patients with childhood dystonia associated with reduced CSF biopterin, responsive to levodopa, and characterized by diurnal and exertional variation are reported from the Developmental and Metabolic Neurology Branch, National Institute of Neurological and Communicative Disorders and Stroke, Bethesda, MD. Of 4 familial cases of dystonia with biopterin deficiency limited to the CNS and of unknown etiology, 2 brothers were more severely affected than 2 sisters, and the dystonia was complicated by hyperreflexia and extensor plantar responses indicative of associated pyramidal tract involvement. The fifth patient had a systemic deficiency of biopterin with hyperphenylalanemia and atypical phenylketonuria, and his dystonic rigidity was purely extrapyramidal and without spasticity. Patients were least symptomatic in the early morning or after a nap and became progressively weak, rigid and dystonic during the day with complete immobility in the afternoon or evening, and often unable to speak or to swallow. Improvement following Sinemet 10/100 began within 36 hours and benefit has been sustained during treatment for 18 
months in 4 patients and 6 years in one, without apparent development of drug tolerance. (Fink JK et al. Dystonia with marked diurnal variation associated with biopterin deficiency. Neurology May 1988; 38:707-711).

COMMENT: The familial cases described here are similar to those with hereditary progressive dystonia reported by Segawa et al and characterized by diurnal variation and extreme sensitivity to levodopa. (Adv Neurol 1976;14:215). Response to levodopa is generally seen in only $10 \%$ of patients with dystonia.

THERAPY FOR TOURETTE SYNDROME

A 12 year-old male with Tourette Syndrome whose symptoms improved with gum chewing is reported from the Division of Pediatric Neurology, Hahnemann University, Philadelphia, PA. With onset at 3 years of age, symptoms had included blinking, head nodding, neck twisting, shoulder shrugs, snorting, sniffing and blowing, and coprolalia. Haloperidol controlled tics but was associated with excessive drowsiness. Clonidine was of some benefit. After chewing gum, using 2-3 sticks to make a large wad, the urge to move and vocalize was decreased and jaw movements made noise-making more difficult. The author recommends gum chewing as an adjunct therapy in Tourette Syndrome to decrease stress, facial movements, and particularly vocalizations. (Brill CB. Gum chewing as therapy for Tourette syndrome. Pediatr Neurol April 1988; $\underline{4}: 128$ ).

COMMENT: Provided that teachers and parents are tolerant of gum chewing, this form of therapy should be recommended. The prevalence of Tourette syndrome was discussed in a previous issue (Ped Neur Briefs March $1988 ; 2: 24)$. Tics were controlled by haloperidol in 13 of 18 children requiring treatment; the disorder was mild and treatment was unnecessary in 23 (56\%) patients (Neurology $1988 ; 88: 472$ ).

\section{LESCH-NYHAN SYNDROME}

Five boys with Lesch-Nyhan syndrome and varying degrees of dystonia, chorea, spasticity, ataxia, dysarthria, and mental retardation were studied at the Depts of Neurology and Medicine, Baylor College of Medicine, and Depts of Neurology and Pediatrics, University of Texas Health Science Center, Houston, TX. Four showed reduction of homovanillic acid (HVA) in the CSF and all had low CSF phenethylene glycol, indicating reducee dopamine and norepinephrine turnover. Three children had high CSF 5-hydroxyindoleacetic acid (HIAA), suggesting increased serotonin turnover. The patient with the most severe chorea had the lowest CSF HVA value, whereas the patient with the least amount of self-mutilation had the highest CSF 5HIAA. Only one patient improved with carbidopa-levodopa, whereas all 5 showed some lessening of self-mutilatory or hyperkinetic behavior in response to tetrabenazine, a monoamine-depleting agent. The study was thought to support the theory of abnormal central monoamine metabolism in Lesch-Nyhan syndrome. (Jankovic J et al. Lesch-Nyhan syndrome: A study of motor behavior and cerebrospinal fluid neurotransmitters. Ann Neurol May 1988; 23:466-469).

COMMENT: Lesch-Nyhan syndrome is an $\mathrm{x}$-linked recessive disorder 